\title{
Triaxial compression and direct shear tests in the parametrization procedure of soil modeled with the Discrete Element Method
}

\author{
Stefan Steidel $^{1, *}$, Jonathan Jahnke ${ }^{1}$, Xiaoyuan Chang ${ }^{1,2}$, Andreas Becker ${ }^{2}$ and Christos Vrettos ${ }^{2}$ \\ ${ }^{1}$ Department Dynamics, Loads and Environmental Data \\ Division Mathematics for Vehicle Engineering \\ Fraunhofer Institute for Industrial Mathematics ITWM \\ Fraunhofer-Platz 1, 67663 Kaiserslautern, Germany \\ e-mail: [stefan.steidel, jonathan.jahnke, xiaoyuan.chang]@itwm.fraunhofer.de \\ 2 Division of Soil Mechanics and Foundation Engineering \\ Technical University of Kaiserslautern \\ Erwin-Schrödinger-Straße, 67663 Kaiserslautern, Germany \\ e-mail: [andreas.becker, christos.vrettos]@ bauing.uni-kl.de
}

\begin{abstract}
Triaxial compression tests as well as direct shear tests are established standardized tests in soil mechanics to characterize and classify different kinds of soil and particularly assess their shear strength in terms of cohesion and angle of internal friction.

In order to model and numerically simulate the behaviour of soil, the Discrete Element Method (DEM) is well-suited and established as a powerful tool. Related activities at Fraunhofer ITWM led to the development of a DEM software suite entitled GRAnular Physics Engine (GRAPE) with the focus to compute and predict the soil's reaction force in the interaction with tools, like a bulldozer blade, a wheel loader or an excavator bucket $[1,2]$. The parametrization procedure for soil in this context relies on data obtained from triaxial compression tests performed in the soil laboratory.

The capability of triaxial compression tests for parametrizing GRAPE has already been shown [3,4]. Due to its comparably high cost and complexity of execution, we herein evaluate direct shear tests regarding accuracy, practicability and robustness as proper complement or alternative.

Within this contribution, we simulate the triaxial compression test and the direct shear test using GRAPE for two different kinds of cohesionless soil, compare the simulation results with experimental data and discuss the suitability of direct shear tests for the parametrization procedure.
\end{abstract}

\section{REFERENCES}

[1] Obermayr, M., Vrettos, C., Eberhard, P.: A Discrete Element Model for Cohesive Soil. Proc. PARTICLES 2013, pp. 783-794 (2013).

[2] Balzer, M., Burger, M., Däuwel, T., Ekevid, T., Steidel, S., Weber, D.: Coupling DEM Particles to MBS Wheel Loader via Co-Simulation. Proc. CVT 2016, pp. 479-488 (2016).

[3] Obermayr, M., Dressler, K., Vrettos, C., Eberhard, P..: Prediction of draft forces in cohesionless soil with the Discrete Element Method. Journal of Terramechanics, Vol. 48, pp. 347-358 (2011).

[4] Jahnke, J., Steidel, S., Burger, M., Papamichael, S., Becker, A., Vrettos, C.: Parameter Identification for Soil Simulation based on the Discrete Element Method and Application to Small Scale Shallow Penetration Tests. Proc. PARTICLES 2019, pp. 332-342 (2019). 